\title{
Crítica, canon y tradición
}

\section{Diálogo con Ricardo González Vigil}

Resumen

El prestigioso y reconocido crítico literario Ricardo González Vigil dialoga con Cuadernos Literarios sobre el panorama de la crítica literaria en el Perú, los aportes de la reciente teoría dentro de los estudios literarios y los escritores al margen del canon.

Palabras claves: teoría literaria, crítica en el Perú, narrativa peruana

Abstract

Prestigious and recognized literary critic Ricardo González Vigil talk whit us about peruvian literary critic's state and the new theory's contributions inside literature studies and non cannon writers.

Key words: literary theory, Peruvian critic, Peruvian narrative

Ricardo González Vigil conversa en esta ocasión con Cuadernos Literarios sobre la actualidad de la crítica y de los estudios literarios en el Perú, la formación del canon y la necesidad de una revisión constante de nuestra tradición.

$\boldsymbol{H}_{\text {periódicos por su columna de crítica }}^{\text {s muy conocida su labor en }}$ de libros. ¿Ha recogido o seleccionado sus artículos en alguna publicación?

Tengo tres recopilaciones de artículos: Retablo de autores peruanos, que apareció en
1990; es una selección de gran parte de mis artículos que recogí y que llegaba hasta César Vallejo. Cerraba así un tomo, pero quedaba fuera una serie de autores posteriores a Vallejo y, como tenía tanto material, se me ocurrió recoger de un lado la vertiente que va hacia lo andino, lo indígena, y algunas 
otras cosas que vienen con la vanguardia, la modernidad, porque la literatura peruana debe ser vista como ambas cosas, no eliminar la una por la otra. Entonces le puse de título: El Perú es todas las sangres, siguiendo el famoso título de Arguedas para representar precisamente la heterogeneidad. Sin embargo, quedaron fuera otros artículos, y el año pasado saqué mediante la editorial San Marcos uno que se llama Años decisivos de la Narrativa Peruana, que recoge autores de la Generación del 50: Ribeyro, Zavaleta, Vargas

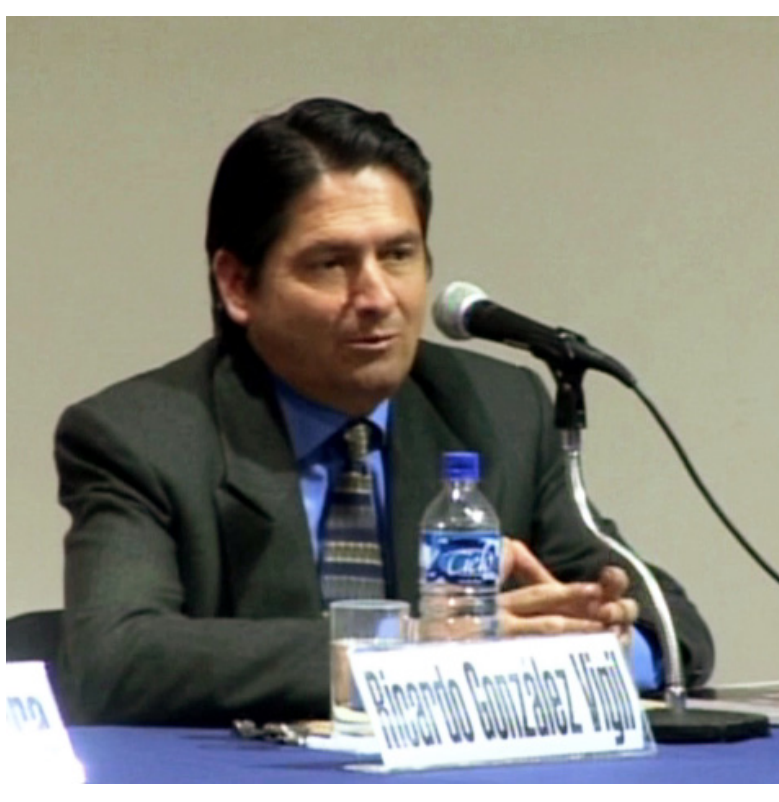
Archivo UCSS
Vicuña, etc., y después autores que aparecen en los años 70, algunos de ellos poetas de la Generación del 60, como Rodolfo Hinostroza o César Calvo, el grupo Trilce de Trujillo, del cual salen Morillo Ganoza, González Viaña y Díaz Herrera. Una figura aislada a la que no le dieron importancia en aquellos años fue Edgardo Rivera Martínez. El grupo de la revista Narración que empezó a publicar en 1966: Miguel Gutierrez, Oswaldo Reynoso, Gregorio Martínez... El material es tanto y mi sueńo es juntar otra gente que aparece a fines de los 60 , pero que no eran del grupo Narración, como Bryce o gente independiente como José Adolph, autores que corresponderían a la generación del 70. En los tres tomos que he publicado hay al principio artículos de carácter más general y algunas precisiones sobre lo que yo creo debe ser la crítica literaria, sobre todo en el volumen de Años decisivos...

Jus t a m e n t e sobre este tema, ya que estamos partiendo de las recopilaciones que usted ha hecho, me gustaría saber qué criterios sigue para preparar sus respectivas antologías. Pienso en este momento en las que preparó sobre el cuento peruano.

Estos criterios los establezco en los prólogos. Sobre el caso de la antología del cuento peruano, salió de una manera no premeditada. Lo que sucedió es que cuando se crea el premio Copé, en la primera versión que correspondía al 79, fui parte del 
jurado y conocí al jefe de relaciones públicas de Petroperú, Pedro Cateriano. A él le gustó el resultado y la acogida que había tenido el concurso, y en una conversación me dijo que hacía tiempo que no había una buena antología de cuentos peruanos. Era cierto, el intento más importante había sido realizado por Alberto Escobar, que ya era antiguo, y había algunas selecciones valiosas pero muy limitadas como por ejemplo la antología de Abelardo Oquendo que había salido por Alianza Editorial. Además tales trabajos se detienen en el 60 o 70. Entonces él me pregunta si yo podría preparar una antología que mostrara el buen momento que estaba pasando el cuento en el Perú. Así nació el proyecto de una antología que abarcara los años previos al Copé. Primero realicé una antología que va del 75 al 79. Él me comentó que le gustó el tomo a los miembros de Petroperú y me animó a realizar los siguientes. Por ello fuimos retrocediendo, hasta que llegué a los dos tomos sobre el origen del cuento peruano y luego preparé una que iba del 80 al 89 y después otra del 90 al 2000. Los prólogos en donde explico con detalle los criterios de mi antología son los que están en el cuento peruano del 75 al 79, y el 68 al 74 que son los primeros en salir, y quizá también en esos dos primeros tomos verdes del origen del cuento peruano. Yo explico que uno puede entender por cuento una forma narrativa muy peculiar, muy específica, si uno tiene un criterio muy rígido, muy preciso de lo que cree que es el cuento. A mí me resultaba un problema: ¿cómo puedes incluir muestras orales, que algunos llamarían mitos, leyendas, fábulas, que no son en el sentido de Poe un cuento?

\section{O también el caso de los cuadros costumbristas}

Exacto, hay variedad de formas breves de carácter narrativo o predominantemente narrativo. Si uno quiere aplicar la idea de cuento que tiene Poe o la definición Cortázar, no facilita las cosas. Por ejemplo, Luis Loayza es muy duro con las tradiciones de Ricardo Palma porque no cumplen con las reglas del cuento: tiene disgresiones, ataca la peculiaridad de Palma como si fuera un desperfecto artístico. Yo creo que más bien es una originalidad dentro de nuestra variedad cultural. El mismo Palma, que conoce las teorías del cuento, afirma que la tradición es una novela en miniatura, así prevé ataques como los que realiza posteriormente Luis Loayza. Yo explico que esta es una antología amplia y dirigida para todo tipo de público y no estrictamente para especialistas. Aclaro en el prólogo que la palabra cuento es la que más entiende la gente y que yo uso el término cuento para significar un relato breve. Así empleo en un sentido muy flexible el término. Sin embargo, establezco distinciones porque la tradición oral, la etnoliteratura, el relato 
mítico-mágico poseen un circuito distinto, el texto no es fijo; hay otra manera de trabajar el relato oral que se diferencia del tipo de cuento en donde el escritor quiere desarrollar un estilo propio y no acepta una modificación del texto. Por eso yo separo siempre en dos grandes partes: la sección de tradición oral y etnoliteratura y la sección de cuentos en el sentido de una literatura especializada. Después, en segundo lugar, está el problema que surge con la teoría literaria más moderna, la cual plantea que el cuento debe ser una obra autónoma, una obra que no sea parte de un conjunto, eso también plantea una serie de problemas. Si bien uno puede pensar en un libro de cuentos completamente autónomo, incluso hay libros en donde los cuentos suponen una correlación entre todos los cuentos, por ejemplo, Huerto Cerrado de Alfredo Bryce Echenique o Tres historias sublevantes de Julio Ramón Ribeyro (si tú lees cada cuento de manera autónoma, no captas todas las conexiones que existen si piensas que es un tríptico). Es tan relativo pensar que un cuento es completamente autónomo. Por otra parte, si uno pretende abarcar todas las formas literarias, te encuentras con textos como Las mil y una noches, El Decamerón, o las novelas, sagas y crónicas que incluyen relatos. Tienes que ser flexible, no manejar una imagen estrecha. Porque es posible sacar un cuento de una crónica del Inca Garcilaso. Ahora, en un sentido estricto, estos relatos no son plenamente autónomos y algunos no son siquiera de ficción en el sentido moderno. Yo preciso esta problemática en los prólogos, porque no he querido dar una imagen rígida que reduciría el cuento a un género que comienza en el Perú a finales del siglo XIX con Luis Benjamín Cisneros, los primeros modernistas, Mercedes Cabello y con los primeros libros de cuentos, en donde encontramos a José Antonio Román y, en el siglo XX, a Clemente Palma. Por otra parte, yo he tenido cuidado al seleccionar pasajes que pertenecen a novelas. El primer caso fue de Manuel Scorza. Yo le dije a Scorza si podía extraer como un relato autónomo la historia de Cecilio Encarnación. Él me autorizó, me dijo que era un cuento que estaba dentro de la novela. Hice lo mismo con la novela de Mario Vargas Llosa, La tía Julia y el escribidor, donde escogí un radioteatro que funcionaba como cuento. En los dos casos conté con el permiso del autor. Entonces, desde el primer tomo se encuentran dos heterodoxias, en donde incluyo dos capítulos o dos partes de novelas que se pueden leer como estructuras en sí mismas, aparte de que adquieren otro valor en el tejido total. Esto fue más claro en el tomo que comprende el lapso 1990-2000. En aquel escogí un capítulo de Ximena de dos caminos de Laura Riesco; lo pude hacer porque esta novela es episódica. También sucedió lo mismo con La destrucción del reino de Miguel Gutierrez, una novela donde 
a partir de unas fotos surgen historias como árboles: un niño contempla una fotografía, la coloca en varios sitios, entonces arma un contrapunto, pero cada capítulo se convierte en una historia autónoma. Me parecería un poco más forzado extraer historias de otras novelas de Gutierrez, y al igual que los anteriores autores estuvo de acuerdo. Yo creo, te vuelvo a repetir, que a veces la gente cree en definiciones escolares fáciles y no es así, la realidad de los textos no es esa simplificación. No estoy tan de acuerdo con los criterios rígidos, y lo digo en los prólogos, porque estoy convencido de que la creatividad literaria es bastante amplia.

\section{Revisando sus artículos, uno puede notar que usted es un entusiasta difusor de la obra de Gamaliel Churata}

Ahora Gamaliel Churata interesa a mucha gente. Es un autor que empecé a leerlo a fines de los años 60 y encontré personas que también estaban interesadas. El propio Luis Alberto Sánchez habla bien de él. Me di cuenta de que Churata era un autor que planteaba un problema de recepción muy grande, porque no maneja la literatura en el sentido rígido que tenemos en esta época. Más se parece a Guamán Poma, debido a que produce unos textos que mezclan todos los discursos: lo lírico, lo narrativo, lo dramático, la filosofía, la historia... así crea un artefacto textual muy libre, a ratos quiere aparecer como una escritura sagrada, como una Biblia, como una enciclopedia. Él dice que es un retablo o que es una especie programa radiofónico. Eso me resulta interesante: una masa textual extraña que Churata produce como una especie de Guaman Poma del siglo XX. Quiero precisar que no creo que el interés de Churata se reduzca a un interés para entender la cosmovisión andina, un interés como documento o como testimonio cultural de multiglosia. También creo que es una obra que toma en cuenta el idioma, pues es el instrumento central en la literatura. Churata tiene una pelea infernal con el idioma. Por eso pienso que hay una hazaña verbal, no solo sociológica. Da una sensación de algo que está en germen o en ciernes, es un borbotón... no te voy a decir que Churata tiene la perfección genial de Vallejo en Trilce, ciertamente no alcanza eso, pero creo que es un gran creador verbal, pues logra una síntesis muy compleja entre el mito y la vanguardia, logra una fusión de elementos entre el pensamiento revolucionario y una apuesta por las raíces. Por ello creo que es un escritor muy importante. Me parece además que, dentro de la propuesta que se conoce como lo real maravilloso americano, él debería ser visto como el primero, incluso temporalmente antes de Miguel Ángel Asturias, Alejo Carpentier, Ciro Alegría y José María Arguedas. En Churata hay una propuesta muy significativa en 
todos los sentidos: literario, idiomático, antropológico, cultural. Riccardo Badini, un especialista italiano que se ha dedicado a estudiar la obra de Churata, está por publicar un libro inédito de Churata, un libro tan grande como $E l$ pez de oro, que se llama Resurrección de los muertos. Como ves, hay una gran cantidad de gente que está estudiando Churata, pues hay numerosos artículos dispersos en revistas. Yo habré ayudado un poco a divulgar el tema en periódicos, pero tenemos trabajos como los de Miguel Ángel Huamán y Manuel Pantigoso, cuyo padre conoció a Churata.

Si bien nuestro canon tiene personajes fácilmente identificables, también tenemosautoresqueexisten nominalmente pero que pasan desapercibidos tanto para los lectores como para los investigadores, como es el caso de Eleodoro Vargas Vicuña.

Él está muchísimo más olvidado, injustamente. Mientras que Churata es un autor que estálleno de calor en la bibliografía, y se avecinan libros, Vargas Vicuña es un autor al cual no se lo ha trabajado con más profundidad, no se lo reedita, no se lo estudia. Yo creo que es uno de los mejores cuentistas de la Generación del 50, tiene una perfección en el lenguaje, una belleza rulfiana, es un prodigio junto con Juan Rulfo. A mí me da una pena porque yo creo que junto a Ribeyro debería mencionarse siempre el nombre de Vargas Vicuña, pues es uno de los mejores cuentistas de esa generación. Pero, claro, Ribeyro tiene una obra más desarrollada, pues él logró ampliar su propuesta.

Otro autor interesante es Carlos Eduardo Zavaleta, quien tampoco recibe la debida atención.

Claro, Zavaleta es el más versátil, ha publicado más que Ribeyro y sigue en plena producción. Yo he escrito mucho sobre él. Tampoco entiendo porque no goza del reconocimiento que merecería. Incluso a nivel nacional no está tan incluido en la enseñanza y fuera del Perú casi no existe. Cuando pienso en los cuentistas del 50 inmediatamente digo Ribeyro, Vargas Vicuńa y luego Zavaleta. Son los cuentistas que a mí me parecen más significativos.

A Zavaleta más bien se le considera importante porque introdujo las lecturas de Joyce, de Faulkner, pero su obra narrativa carece de resonancia.

Exacto, lo han ido reduciendo a eso. Yo, por ejemplo, incluso hehecho notar en mi edición de Los ríos profundos que Arguedas mediante cartas le pide a Manuel Moreno Jiménez que le mande libros norteamericanos. Eso demuestra que Arguedas lee a Faulkner 
"Es bueno que siempre se realicen revisiones criticas, nuevas antologias, de esa manera se logra rescatar autores que no recibieron atención. Es necesario que cada generación, que cada nuevo grupo relea la tradición literaria para ir afinando errores, lagunas, descuidos»

en 1941, por lo que podríamos decir que hay un conocimiento de Faulkner antes de Zavaleta. Además, el interés por Joyce en nuestro contexto comenzó en los años 20. Entonces relativizaría yo esa afirmación. Pero más importante que eso es que es un escritor muy valioso, más en el cuento que en la novela, aunque tiene una novela formidable que se llama Pálido pero sereno. Él maneja más el relato breve y la novela corta, como Los Ingar, y sabe retratar el mundo rural y el mundo urbano. Es un autor con una narrativa muy compleja.

\section{Lo último que leí de él fue $E l$ Padre del Tigre}

Es el texto vinculado con la subversión y llega hasta las experiencias actuales: los cambios de Lima y de la sierra. Es un hombre que junta las dos cosas: la parte andina y la parte criolla. No ha caído en simplificaciones, siempre ha creído más en un Perú complejo, ha defendido trascender la diferencia, ha hablado más de un Perú integrado. No entiendo por qué hay varios escritores que teniendo méritos no logran el reconocimiento, no entran a los circuitos, ¿es cuestión de vínculos, de agentes editoriales? No sé. Es cierto que el reconocimiento es un factor que a la larga no va a ser el importante, pero hace sufrir a los autores mientras viven, porque un autor cuando es bueno 100 o 200 años después se impone; por ejemplo, Churata que fue ignorado tanto tiempo y murió como si no existiera, y ahora se ha despertado el interés por su obra.

Lo mismo también ha pasado con Oquendo de Amat que en lo últimos años ha alcanzado cierta resonancia mediática. Y que ahora entusiasma tanto porque es un gran poeta. El éxito está librado a tantos factores; por eso, es bueno que siempre se realicen revisiones críticas, nuevas antologías, de esa manera se logra rescatar autores que no recibieron atención. Es necesario que cada generación, que cada nuevo grupo relea la tradición literaria para ir afinando errores, lagunas, descuidos.

Otro autor que si bien fue importante durante su tiempo, pero que ahora no 
recibe mucha atención, es José Santos Chocano en los círculos académicos.

Yo siempre he considerado que se ha cometido una injusticia con Chocano. Desde hace treinta años he hecho comentarios sobre ello, los cuales están recogidos en Retablo de autores peruanos. Incluso, me acuerdo que comenté una reedición de Aladino, o la vida y obra de José Santos Chocano, de Luis Alberto Sánchez, cuando comentaba en Correo. A ese comentario le puse de título «La poética de Chocano». La persona encargada de la sección cultural modificó el título, cosa que se puede hacer porque se juzga que el título no es periodístico, y le puso "Chocano tolerable». Yo me fastidié, porque era una imagen que mostraba esa recepción cultural que hay de Chocano. Como yo pedía una lectura más equlibrada, lo juzgaron como tolerable.

Yo creo que en el caso de Chocano han influido dos cosas: una es el desprestigio de su evolución ideológica, porque después de haber sido un hombre a favor del cambio, simpatizante de González Prada, de la Revolución Mexicana, se fue convirtiendo en amigo de dictadores, en un hombre de ultraderecha que llega incluso a cometer un asesinato donde la víctima fue un joven periodista. Además su comportamiento era muy desagradable. Entonces, estos factores extrapoéticos le fueron restando audiencia. Por otro lado, cuando empezaron a realizarse estudios de mayor profundidad como los de Estuardo Nuñez y Luis Monguió, o los artículos de Jorge Basadre, estos autores estaban movidos por un buen objetivo, que era reconocer la mayor sutileza y calidad poética de Eguren y de Vallejo, por comparación se subrayó las carencias de Chocano. Quizá lo que se ha practicado mucho en la crítica literaria peruana ha sido comparar a un escritor que tuvo éxito con otro que no, para reconocer finalmente a este último como si fuera una especie de justicia tardía. Para poner las cosas en su sitio, al hacer la comparación se genera la percepción de que si uno reconoce a Eguren entonces tiene que negar a Chocano. Un poco también es lo que le ha pasado entre Ciro Alegría y José María Arguedas. Yo pienso que es injusto. Una cosa es que uno admita la mayor genialidad de Eguren y de Vallejo, pero de allí a que Chocano no interese, no es justo. Si uno compara a Chocano con los poetas modernistas de otros países, nota que estos son menos, pero los estudian, caso de Amado Nervo, Leopoldo Lugones, José Asunción Silva que son autores también con limitaciones. Tampoco ellos son Rubén Darío, un poeta que ciertamente supera a todos los poetas modernistas. Chocano es importante, incluso reconociendo sus limitaciones; por ello Washington Delgado dijo, con toda razón, que es el primer poeta peruano que propone una poética, que desarrolla una 
propuesta propia, que tiene un lenguaje, un estilo y, por ende, es influyente en toda la poesía hispanoamericana. Yo creo que no puede faltar en una antología de la poesía peruana. Pero en algunas lo excluyen y eso no puede ser.

\section{¿Cómo observa el estado actual de la crítica en el Perú?}

En primer lugar, habría que precisar, cuando se usa la palabra crítica hay una posibilidad de entender la crítica en el sentido más peculiar, más propio de la crítica. La crítica implica un acto de juicio valorativo, separar la paja del grano o ejercer el criterio para distinguir. Entonces un crítico si no juzga, si no arriesga, no es crítico, eso si lo tomamos en el sentido más estricto. Pero a veces se utiliza la palabra crítico en general para referir a alguien que estudia la literatura. Hay gente que estudia la literatura y evita los juicios valorativos, realiza más bien un trabajo académico, exegético, acumula información. Son eruditos, hacen discusiones teóricas y creen que la valoración es un elemento tan subjetivo que depende de tantos factores que no es propio de un estudio serio. Es decir, quieren hacer ciencia de la historia literaria. Si uno quiere hacer ciencia literaria será un especialista, un gran conocedor del tema. Sin embargo, creo que también es posible hacer un juicio dentro de una obra especializada, como es el caso de Dámaso Alonso cuando reinvindica el valor de Góngora. Por otro lado, otra percepción que hay sobre el crítico es que se piensa que un crítico debe ser un ensayista, es decir, una persona que, a partir de la lectura de un autor plantea toda una meditación, como intenta Vargas Llosa en Historia de un Deicidio a partir de su lectura de Gabriel García Márquez o en La tentación de lo imposible cuando lee a Víctor Hugo. Aquí, en el Perú, Vargas Llosa, Miguel Gutierrez, Luis Loayza y también Julio Ramón Ribeyro han practicado este tipo de crítica reflexiva, de vuelo, que marca un poco más. Mucha gente cree que eso es hacer crítica y se piensa en autores como Eric Auerbach, Ernst Curtius,

"Hay gente que estudia la literatura y evita los juicios valorativos, realiza más bien un trabajo académico, exegético, acumula información. Son eruditos, hacen discusiones teóricas y creen que la valoración es un elemento tan subjetivo que depende de tantos factores que no es propio de un estudio serio» 
George Steiner. Incluso hay filósofos que intentan hacer crítica, como Ortega y Gasset que realiza Las meditaciones del Quijote, o el caso más significativo de Nietszche que escribe El origen de la tragedia y propone un sistema filosófico donde expone su teoría de lo dionisíaco y lo apolíneo. Sin embargo, yo siempre digo burlonamente que a mí Nietszche me sirve muy poco para entender la tragedia griega. Claro que me sirve mucho para entender al ser humano, la lucha interna que hay en su propia naturaleza, pero no

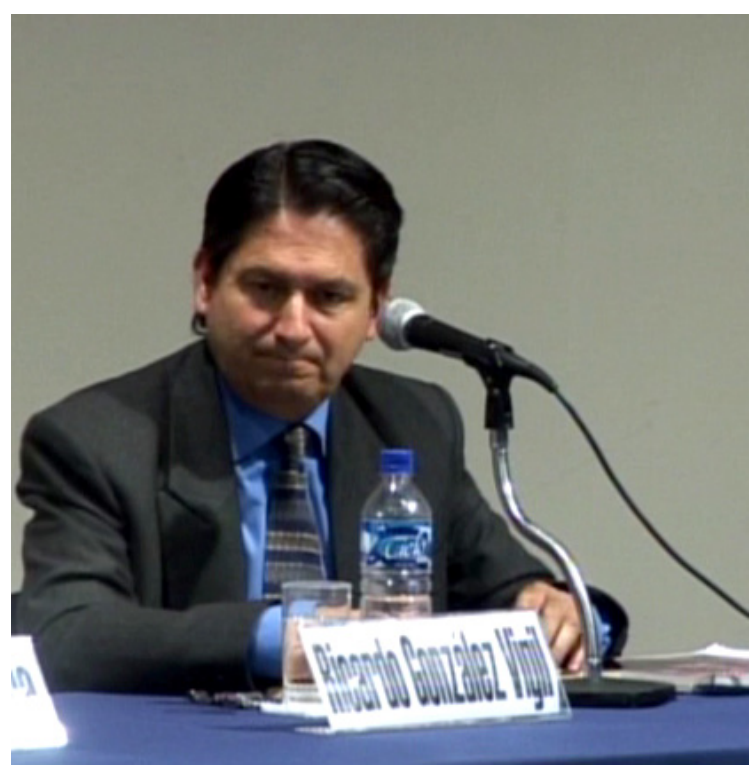
Archivo UCSS al cual me dirijo. Si uno escribe a un público no especializado, es necesario orientarlo. Para tal fin, tengo que combinar lo didáctico y lo valorativo. Didáctico: uso todos mis conocimientos para que el lector comprenda y, además, allí está el riesgo, ejerzo el criterio. Con ese balance, yo te diría que con todas las limitaciones, el Perú tiene una tradición crítica. Desde el punto de vista de la reflexión teórica y retórica, esta tradición empieza con el Discurso en loor de la poesía de Clarinda. En el caso de lo valorativo podemos me da materiales para entender el texto, me sirven más otros trabajos filológicos... Soy consciente, planteo las cosas como lo complejas que son, porque yo tengo la suerte de ser profesor de la universidad, de haber realizado trabajos eruditos como mis ediciones críticas de Vallejo. Y, de otro lado, tengo la obligación de opinar inmediatamente sobre un libro que acaba de aparecer, y me arriesgo a dar una valoración. Entonces, yo juego entre lo académico y la divulgación, y trato de pensar en el público empezar con el Apologético... del Lunarejo. Como se puede notar, tenemos los mejores antecedentes coloniales, a nivel americano, para una reflexión sobre la poesía o una actitud valorativa. Quizá lo que ha habido mucho es historiadores de la cultura como José de la Riva Agüero, Raúl Porras Barrenechea, Jorge Basadre, el propio Luis Alberto Sánchez, que estudia la literatura como un derrotero para entender la historia cultural del Perú. Hay un tipo de visión historiográfica que ha dado nombres como 
Alberto Tauro, Aurelio Miro Quesada, Tamayo Vargas. Por otro lado, también tenemos filólogos como Luis Jaime Cisneros y Armando Zubizarreta. Desde los años 50 hacia adelante tenemos especialistas que realizan buenas ediciones de textos. También muchos se han interesado por la discusión teórica y metodológica, por intentar una teoría aplicada a nosotros, como Antonio Cornejo Polar o Enrique Ballón. Asimismo, surgen más actividades propiciadas por los jóvenes, como encuentros, coloquios y la publicación de revistas. Yo sí creo que hay un discurso crítico sobre la literatura peruana, el cual también ha generado muchas polémicas. Somos una de las áreas que más discutimos, para nosotros la literatura suscita pasiones.

Actualmente el marco teórico para realizar un análisis de los textos literarios es bastante amplio: teorías de género, psicoanálisis, estudios culturales... En nuestro contexto, ¿cree que hay trabajos consistentes en la aplicación de estos modelos para analizar nuestra literatura?

A nivel de tesis universitarias y ponencias existe una inquietud teórica. Yo creo que el riesgo de esas posturas es no darse cuenta de que es una manera de leer que no agota la obra. Bien aplicado, con rigor, es un aporte iluminador. Por ejemplo, yo terminé una amplia antología de la literatura peruana de mujeres, en la cual incluyo noventa poetas. Mediante esta antología estoy tratando de ayudar a que se les dé a estas poetas el sitio que merecen. Por diversas razones no se ha investigado suficientemente en la producción de mujeres. Es más frecuente que la mujer no publique libros, así su obra ha quedado inédita o dispersa, por ello tuve que investigar más en revistas y en periódicos. También tuve que revisar los aportes del feminismo, que aunque puede caer en excesos, en parte tiene razón, porque hay un concepto de lo femenino que es una construcción cultural.

Asimismo, en el contexto más reciente, también existe un interés en el discurso de la violencia a razón del conflicto político y militar que padecimos.

El tema de la violencia, que siempre ha existido en nuestro país pero que se ha vuelto muy fuerte en los años 80 y 90, crea un clima que hay que saber procesar, que exige un trabajo interdisciplinario, no se puede realizar desde una perspectiva reducida. Me parece que nos es difícil procesar eso, porque no se ha logrado del todo situar las causas del conflicto, y se siguen manejando elementos que nos dividen, que nos llevan a posturas ideológicas contrapuestas. Es demasiado palpitante el problema. Antes era más complicado hablar sobre ello, como hicieron, en los años 80 , los cuentos 
de Luis Nieto Degregori o la poesía de Marcial Molina en Ayacucho. En aquel tiempo corrías el riesgo incluso de que te consideren subversivo o que los subversivos te mataran porque no les gustaba tu versión. El informe de la comisión de la verdad ha creado un cuadro, una referencia. Tenemos una literatura que se hizo durante los años convulsos y ahora, en esta década, hay más elementos de juicio y distancia que contribuyen a la producción de literatura referida a este tema. 